\title{
Intermittent hypoxia-activated cyclooxygenase pathway: role in atherosclerosis
}

\author{
Elodie Gautier-Veyret ${ }^{1,2}$, Claire Arnaud¹, Magnus Bäck ${ }^{3}$, Jean-Louis Pépin ${ }^{1,4,5}$, \\ Marcelo H. Petri ${ }^{3}$, Jean-Philippe Baguet ${ }^{4,6}$, Renaud Tamisier ${ }^{1,4,5}$, Patrick Lévy ${ }^{1,4,5}$ \\ and Françoise Stanke-Labesque ${ }^{1,2,4}$
}

Affiliations: ${ }^{1}$ INSERM, U1042, Grenoble, ${ }^{2} \mathrm{CHU}$, Hôpital A. Michallon, Laboratoire de Pharmacologie, BP217, Grenoble, ${ }^{4}$ Université Grenoble 1, Faculté de Médecine, UFR1, Grenoble, ${ }^{5} \mathrm{CHU}$, Hôpital A. Michallon, Pôle rééducation et Physiologie, BP217, Grenoble, ${ }^{6} \mathrm{CHU}$, Hôpital A. Michallon, Clinique de Cardiologie, BP217, Grenoble, France, ${ }^{3}$ Dept of Medicine, Karolinska Institute and University Hospital, CMM L8, 03, Stockholm, Sweden.

Correspondence: F. Stanke-Labesque, Laboratory of Pharmacology, Grenoble University Hospital, BP 217. F-38043 Grenoble Cedex 9, France. E-mail: FStankedachu-grenoble.fr

ABSTRACT Intermittent hypoxia, the main stimulus of obstructive sleep apnoea (OSA), induces inflammation, leading to early atherosclerosis. Whether the cyclooxygenase (COX) pathway contributes to intermittent hypoxia-induced atherosclerosis remains to be determined.

We studied the effects of 8-weeks of intermittent hypoxia exposure on COX-pathway gene expression and atherosclerosis, and the influence of COX-1 inhibition by SC-560 on atherosclerosis progression in aortas of apolipoprotein $\mathrm{E}^{-/-}$mice. Urinary 11-dehydrothromboxane $\mathrm{B}_{2}\left(11-\mathrm{dTXB}_{2}\right)$ was assessed in 50 OSA subjects free of cardiovascular risk factor matched for age and body mass index with 25 controls, and 56 OSA with cardiovascular risk factor.

Intermittent hypoxia significantly increased atherosclerotic lesion sizes, mRNA levels of COX-1 and thromboxane synthase (TXBS). Lesion sizes correlated to COX $-1(r=0.654, p=0.0003)$ and TXBS $(r=0.693$, $\mathrm{p}<0.0001)$ mRNA levels. COX-1 inhibition reduced lesion progression in intermittent hypoxia mice only $(\mathrm{p}=0.04)$. Urinary 11- $\mathrm{dTXB}_{2}$ was similar in OSA subjects free of cardiovascular risk factor and controls, but was increased by $13 \%(p=0.007)$ in OSA subjects with cardiovascular risk factor compared with those without.

Although OSA itself was not associated with increased urinary 11-dTXB 2 concentration, the COX-1 pathway was activated in intermittent hypoxia-exposed mice and in OSA subjects presenting with cardiovascular risk factor, and may contribute to intermittent hypoxia-induced atherogenesis. COX-1 inhibition could be of clinical interest in the prevention of cardiovascular morbidity in OSA.

@ERSpublications

COX-1 inhibition could be of clinical interest in the prevention of cardiovascular morbidity in obstructive sleep apnoea http://ow.ly/lUIb5

This article has supplementary material available from www.erj.ersjournals.com

Received: June 182012 | Accepted after revision: Sept 262012 | First published online: Oct 112012

Clinical trial: The study is registered at www.clinicaltrials.gov with identifier number NCT01089257.

Support statement: This study was supported by a grant from "le vivier de la recherche médicale" from Grenoble University of Medicine, PHRC 2010, ResMed Foundation, Mairie de Paris ("Research in Paris"), The French-Swedish Foundation and the Swedish Heart and Lung Foundation.

Conflict of interest: None declared.

Copyright @ERS 2013 


\section{Introduction}

Obstructive sleep apnoea (OSA) is characterised by recurrent episodes of partial or complete upper airway obstruction occurring during sleep, leading to chronic intermittent hypoxia (CIH). This hallmark is the main factor involved in cardiovascular remodelling in OSA [1]. In OSA patients, early signs of atherosclerosis correlated to hypoxia severity [2], even after adjustment for confounding factors. OSA is associated with increased cardiovascular morbidity and mortality, and is identified as an independent cardiovascular risk factor (CVRF) [3]. Moreover, exposure of apolipoprotein E-deficient $\left(\mathrm{ApoE}^{-/-}\right) \mathrm{mice}^{2}$ [4, 5] and $\mathrm{C} 57 \mathrm{BL} / 6 \mathrm{~J}$ mice [6] to $\mathrm{CIH}$ accelerated atherogenesis progression.

Atherosclerosis is a chronic inflammatory disease. Among the many inflammatory mediators involved in atherogenesis, we previously demonstrated the contribution of arachidonic acid-derived metabolites in OSA patients. Notably, OSA patients exhibited elevated levels of leukotriene $\mathrm{B}_{4}[7,8]$ and cysteinyl-leukotrienes [9] that were associated with vascular remodelling. However, the cyclooxygenase (COX)-dependant pathway of arachidonic acid metabolism was poorly studied in OSA patients, and its implication in $\mathrm{CIH}$ induced atherogenesis is unknown.

Thromboxane $\mathrm{A}_{2}\left(\mathrm{TXA}_{2}\right)$ and prostacyclin $\left(\mathrm{PGI}_{2}\right)$ are two COX-derived metabolites of arachidonic acid metabolism. TXA 2 is predominantly generated by platelets through COX type 1 isoform (COX-1) and thromboxane synthase (TXBS). TXA 2 is quickly metabolised to thromboxane $\mathrm{B}_{2}\left(\mathrm{TXB}_{2}\right)$ and 11dehydrothromboxane $\mathrm{B}_{2}\left(11-\mathrm{dTXB}_{2}\right)$, two metabolites respectively quantifiable in plasma and urine. $\mathrm{TXA}_{2}$ binding on its TP receptors induces platelet activation, vasoconstriction, vascular smooth muscle cell proliferation and increases expression of adhesion molecules [10]. The production of $\mathrm{PGI}_{2}$ mainly depends on endothelial COX-2 and prostacyclin synthase (PGIS). PGI $_{2}$ is rapidly metabolised to 6-keto-prostaglandin F1 $\alpha$ (6-keto-PGF1 $\alpha$ in plasma and 2,3-dinor-6-ketoprostaglandin F1 $\alpha$ (PGI-M) excreted in urine. In contrast to $\mathrm{TXA}_{2}, \mathrm{PGI}_{2}$ binding on its IP receptors inhibits platelet aggregation and vasoconstriction, and reduces chemotaxis and expression of adhesion molecules [10]. Thus, $\mathrm{TXA}_{2}$ and $\mathrm{PGI}_{2}$ have antagonist properties.

A recent growing body of evidence suggests a major role of the COX pathway in the pathogenesis and progression of atherosclerosis. In fact, pharmacological inhibition of COX pathway [11, 12] or genetic deletion $[13,14]$ delayed atherosclerosis in different mouse models of atherosclerosis. Furthermore, the urinary 11- $\mathrm{dTXB}_{2} / \mathrm{PGI}_{2}-\mathrm{M}$ ratio is enhanced in elderly subjects with past history of serious cardiovascular event (stroke and myocardial infarction) [15]. As selective COX-2 inhibitors may have nonfavourable effects on this ratio and increase cardiovascular risk [16], the role of COX-1 inhibition has received less attention. In addition, data in OSA are very limited and conflicting, showing either an increased [17] or a decreased [18] urinary ratio $11-\mathrm{dTXB}_{2} / \mathrm{PGI}_{2}-\mathrm{M}$. Thus, the aim of the present study was to characterise the COX pathway in $\mathrm{ApoE}^{-/-}$mice exposed to $\mathrm{CIH}$ and in OSA patients, and to investigate its link with $\mathrm{CIH}$ induced atherogenesis and OSA-associated early vascular remodelling.

\section{Materials and methods}

\section{Experimental animal study}

Male ApoE ${ }^{-/-}$mice (14 weeks old) were purchased from the Charles River Laboratories (L'Arbresle, France). All animal procedures were conducted in accordance with the European Convention for the Protection of Vertebrate Animals used for Experimental and Other Scientific Purposes (Council of Europe, European Treaties ETS 123, Strasbourg, March 18, 1986) and to the Guide for the Care and Use of Laboratory Animals (NIH Publication no. 85-23, revised 1996).

In a first series of experiments, mice were randomised to 8 weeks of $\mathrm{CIH}$ (cyclic 21-25\% inspiratory oxygen fraction $\left(\mathrm{FIO}_{2}\right), 60$-s cycle for $8 \mathrm{~h} \cdot \mathrm{day}^{-1}$ ) or normoxic air as previously described ( $\mathrm{n}=15$ in each group) [1]. In a second series of experiments, mice were exposed to 8 weeks of $\mathrm{CIH}$ or normoxic air $(\mathrm{n}=20$ in each group), and randomised to receive either placebo or the selective COX-1 inhibitor SC-560 (Interchim, Montluçon, France) (15 mg $\cdot \mathrm{kg}^{-1}$ daily [12]) in their food, for the last 4 weeks of exposure. All mice were fed with a normal diet, ad libitum, during all experiments.

At the end of exposure, blood was collected under anaesthesia (ketamine/xylazine $100 \mathrm{mg} \cdot \mathrm{kg}^{-1} / 10 \mathrm{mg} \cdot \mathrm{kg}^{-1}$ by intraperitoneal injection) for haematocrit and lipid measurements. Entire aortas were harvested. Abdominal aortas were placed in RNAlater (Life Technologies, Villebon-sur-Yvette, France), frozen in liquid nitrogen and stored at $-80^{\circ} \mathrm{C}$ until analysis. Immediately after their sampling, thoracic aortas were placed in Tyrode solution ( $137 \mathrm{mM} \mathrm{NaCl}, 2.7 \mathrm{mM} \mathrm{KCl}, 0.41 \mathrm{mM} \mathrm{NaHPO}, 2 \mathrm{mM} \mathrm{CaCl}, 5 \mu \mathrm{M} \mathrm{MgCl}_{2}$, $11.9 \mathrm{mM} \mathrm{NaHCO}_{3}$ and $5.5 \mathrm{mM}$ glucose) for prostanoid secretion measurements.

\section{Atherosclerotic lesion size quantification}

Atherosclerotic lesions of aortic roots were studied by Oil-Red-O (Sigma Aldrich, Saint Quentin-Fallavier, France) staining, as previously described [5]. For each aorta, lipid deposition was quantified from five 
sections $(8-\mu \mathrm{m}$ thickness), separated by $80 \mu \mathrm{m}$ from each other, using computer image analysis (NisElement; Nikon Instruments Inc., Melville, NY, USA).

\section{Aortic secretion of prostanoids}

Thoracic aortas were incubated for $15 \mathrm{~min}$ in Tyrode solution maintained at $37^{\circ} \mathrm{C}$, aerated with $95 \% \mathrm{O}_{2}$ and 5\% $\mathrm{CO}_{2}$, in presence of calcium ionophore A23187 (Sigma Aldrich, Saint Quentin Fallavier, France) at $10^{-6} \mathrm{M}$. Supernatants were immediately frozen at $-80^{\circ} \mathrm{C}$ for prostanoid measurement, while aortas were dried for measurement of dry tissue weight.

\section{COX-pathway gene expression}

Total mRNA was isolated from aorta using the RNeasy kit (Qiagen, Hilden, Germany) as previously described [19] and reverse-transcribed using Superscript II (Invitrogen, Carlsbad, CA, USA) with random hexamers according to the manufacturer's instructions. Quantitative TaqMan PCR was performed on a 7900 HT using primer/probe pairs designed with Assay-On-Demand (both Applied Biosystems; Life Technologies SAS, Saint Aubin, France) (online supplementary table S1). Data were normalised to $18 \mathrm{~S}$ ribosomal protein mRNA and expressed as $2^{-\Delta \mathrm{CT}}$.

\section{Clinical study}

The local ethics committee approved this study according to the Declaration of Helsinki. All participants gave written informed consent.

113 newly diagnosed OSA patients were consecutively entered into the study between January 2007 and April 2011, as well as 25 controls. Patients were referred to the sleep laboratory of Grenoble University Hospital for symptoms suggesting OSA. Exclusion criteria were past history of stroke or myocardial infarction, known hypertension, and treatment with nonsteroidal anti-inflammatories, aspirin, steroids, antidiabetic, antihypertensive and lipid-lowering drugs.

\section{Design}

The specific contribution of $\mathrm{CIH}$ on $11-\mathrm{dTXB}_{2}$ urinary concentrations was assessed in 25 controls carefully matched for age and body mass index (BMI) with 50 nonobese OSA patients (i.e. one healthy subject for two OSA subjects). All subjects were free of any known CVRFs. A sample size of 50 OSA patients and 25 controls was calculated as adequate to show an increase of $30 \%$ in urinary $11-\mathrm{dTXB}_{2}$ excretion with at least $90 \%$ power and at the $5 \%$ significance level. This sample size calculation was based on preliminary data obtained on healthy subjects showing a mean concentration of $11-\mathrm{dTXB}_{2}$ of $647 \mathrm{pg} \cdot \mathrm{mL}^{-1}$ with a standard deviation of $287 \mathrm{pg} \cdot \mathrm{mL}^{-1}$. As the dispersion of $11-\mathrm{dTXB}_{2}$ was higher in patients with cardiovascular disease than in controls, we chose a mixed model including one healthy for every two OSA subjects.

The influence of CVRFs (which comprise obesity (BMI $>30 \mathrm{~kg} \cdot \mathrm{m}^{-2}$ ); hypertension (clinical diastolic blood pressure $(\mathrm{BP})>90 \mathrm{mmHg}$ and systolic $\mathrm{BP}>140 \mathrm{mmHg}$ ); dyslipidemia (low-density lipoprotein (LDL) cholesterol $>4.13 \mathrm{mmol} \cdot \cdot^{-1}$ or association of total cholesterol $>5.16 \mathrm{mmol} \cdot \mathrm{l}^{-1}$ and high-density lipoprotein (HDL) cholesterol $<1.03 \mathrm{mmol} \cdot \mathrm{l}^{-1}$ ); smoking; and metabolic syndrome (defined by criteria of International Diabetes Federation [20])) on urinary excretion of $11-\mathrm{dTXB}_{2}$ was evaluated in the whole cohort of 113 OSA subjects. Patients were stratified on the presence or not of one of these CVRFs.

The influence of continuous positive airway pressure (CPAP) on urinary 11- $\mathrm{dTXB}_{2}$ concentration was studied in 14 OSA subjects free of any CVRF and 21 OSA patients with CVRF(s) who were adherent to CPAP. CPAP adherence was defined as CPAP daily use for $>4 \mathrm{~h}$ [9].

All subjects underwent an overnight polysomnography as described [9]. Sleep apnoea was defined as an apnoea/hypopnoea index (AHI) $\geqslant 5$ events $\cdot \mathrm{h}^{-1}$ of sleep and symptoms or respiratory disturbance index (RDI), including flow limitation episodes $>15$ events $\cdot \mathrm{h}^{-1}[21]$.

Urine sample for $11-\mathrm{dTXB}_{2}$ quantification and venous blood for biochemical measurements were collected at $07: 00 \mathrm{~h}$ at the end of the nocturnal polysomnographic recordings and were stored at $-80^{\circ} \mathrm{C}$ until later analysis.

Carotid ultrasonography was performed in 97 OSA patients and 24 controls as previously described [2].

Biological measurements

Plasma cholesterol, triglycerides, glucose and high-sensitivity C reactive protein (hsCRP) concentrations were determined using enzymatic colorimetric methods on a Dimension Vista analyser (Siemens AG, Erlangen, Germany). LDL cholesterol was calculated using the Friedewald formula (total cholesterol-HDL 
cholesterol-(triglycerides/5)). Urinary $11-\mathrm{dTXB}_{2}$ of patients, $\mathrm{TXB}_{2}$ and 6-keto-PGF- $1 \alpha$ in supernatants of mice were measured by liquid chromatography-tandem mass spectrometry (LC-MS/MS) as previously described $[9,22]$. The limits of quantitation of $\mathrm{TXB}_{2}$ and 6 -keto-PGF1 $\alpha$ were $14 \mathrm{pg} \cdot \mathrm{mL}^{-1}$ and $7.25 \mathrm{pg} \cdot \mathrm{mL}^{-1}$, respectively, and their inter- and intra-assay coefficients of variation were $<12 \%$ for both analytes.

\section{Statistical analysis}

Statistical analyses were performed using NCSS97 (Kaysville, Utah) or SAS (SAS 9.1, Cary, NC, USA) for the mixed model. Data were expressed as median and 10th and 90th percentiles. Normal distribution was tested using Kolmogorov-Smirnov nonparametric test. Comparisons between normoxic air and CIH mice were made using the t-test. Comparisons between OSA patients and controls matched for age and BMI (one control for two OSA subjects) were made using a mixed model. Noncontinuous variables were compared using Fisher's test. Comparisons between more than three groups were made using the Kruskal-Wallis test and subsequent pairwise comparisons were made with the Bonferroni multiple-comparison test. For patients treated by CPAP, differences between baseline and post-CPAP values were analysed using a paired t-test or the Wilcoxon signed-rank test. Relationships between two variables were studied with single regression. A multiple linear regression analysis was performed, taking into account the variables that correlated with dependant variable urinary $11-\mathrm{dTXB}_{2}$ in humans. A p-value of $<0.05$ was considered significant.

\section{Results}

\section{Experimental animal study}

Weight, lipid levels and haematocrit

After 8-weeks of exposure, bodyweight and plasma cholesterol levels were similar in $\mathrm{CIH}$ and normoxic air mice (table 1). Haematocrit was significantly higher in $\mathrm{CIH}$ mice compared with normoxic air mice (table 1).

\section{Atherosclerotic lesion sizes}

Atherosclerotic lesions on aortic roots were higher $(\mathrm{p}=0.008)$ in $\mathrm{CIH}$ mice compared with normoxic mice (CIH 66532 (32741-163224) versus normoxic air 37675 (6798-75596) $\left.\mathrm{mm}^{2} ; \mathrm{p}=0.03\right)$. Lesion sizes correlated with plasma total cholesterol in normoxic air mice $(\mathrm{r}=0.501, \mathrm{p}=0.04)$ but not in $\mathrm{CIH}$ mice.

COX-pathway gene expression

COX-1 and TXBS aortic mRNA levels were significantly increased in CIH group compared with normoxic air, whereas COX-2, PGIS, TP and IP receptors aortic mRNA levels were not significantly different between both groups (fig. 1a). mRNA levels of COX-1 and TXBS significantly correlated with lesion sizes (fig. 1b).

Aortic prostanoid secretion

A23187-stimulated 6-keto-PGF1 $\alpha$ and $\mathrm{TXB}_{2}$ secretions and 6-keto-PGF1 $\alpha / \mathrm{TXB}_{2}$ ratio were similar in aorta from $\mathrm{CIH}$ and normoxic air mice (table 2). 6-keto-PGF1 $\alpha$ and $\mathrm{TXB}_{2}$ concentrations were highly correlated $(\mathrm{r}=0.842, \mathrm{p}<0.0001)$.

\section{Effects of COX-1 inhibition}

In both normoxic air and CIH groups, treatment with SC-560 significantly decreased $\mathrm{TXB}_{2}$ and 6-ketoPGF1 $\alpha$ aortic secretion versus placebo (table 2). There was no difference between groups for total cholesterol and bodyweight (table 2). Treatment with SC-560 significantly reduced lesion size by $35 \%$ in $\mathrm{CIH}$ mice whereas it had no effect in normoxic air mice (fig. 2).

TABLE 1 Bodyweight, plasma cholesterol level and haematocrit of apolipoprotein E-deficient $\left(\mathrm{ApoE}^{-/-}\right)$mice exposed for 8 weeks to chronic intermittent hypoxia $(\mathrm{ClH})$ or normoxia

\begin{tabular}{lccc} 
& Normoxia & CIH & p-value \\
\hline Subjects $\mathbf{n}$ & 15 & 15 & \\
Bodyweight after exposure & $31.7(28.5-33.9)$ & $30.3(27.8-32.6)$ & 0.08 \\
Total cholesterol mmol. $\mathrm{L}^{-1}$ & $12.2(5.24-18.7)$ & $14.6(7.10-19.1)$ & 0.46 \\
Haematocrit \% & $45.0(41.4-47.0)$ & $47.0(44.2-55.0)$ & $\mathbf{0 . 0 0 6}$ \\
\hline
\end{tabular}

Data are presented as median (10th-90th percentiles). 

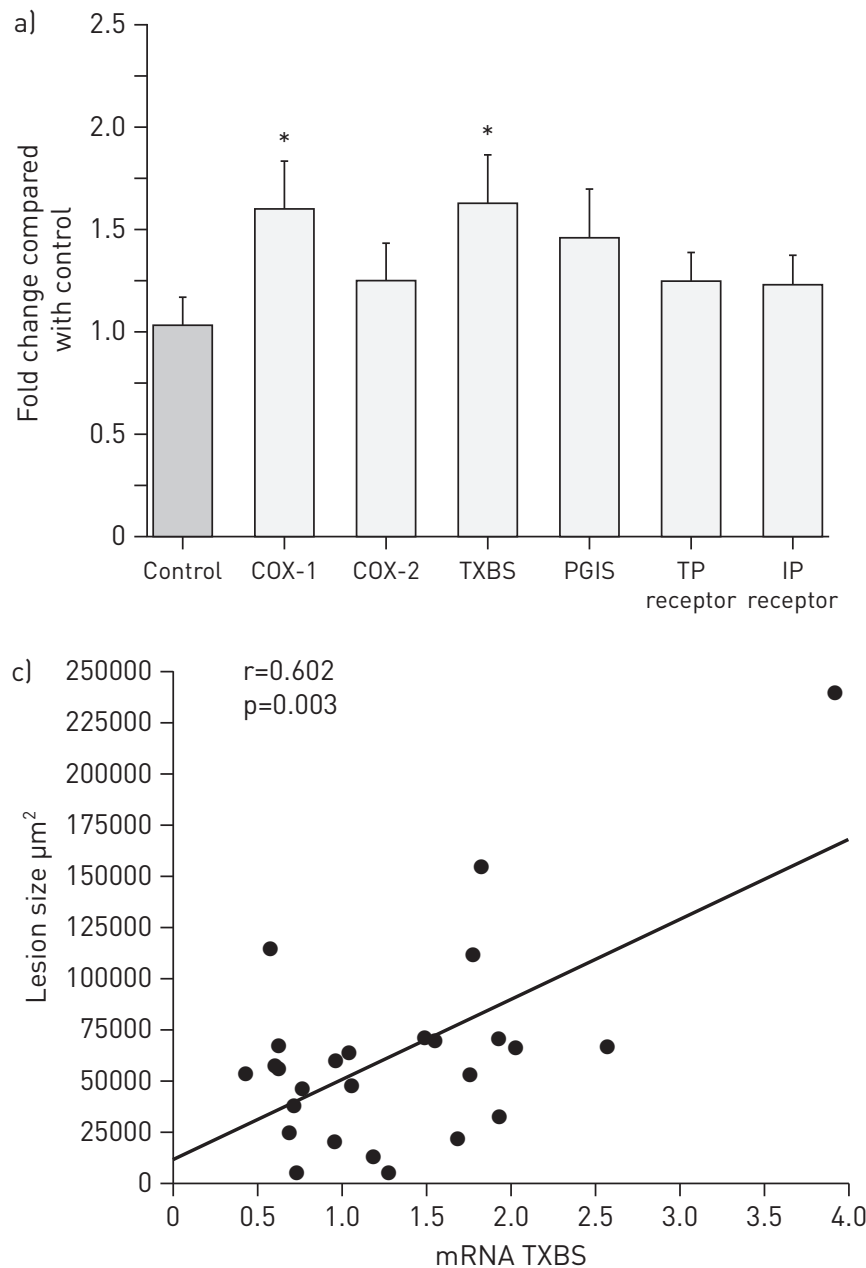

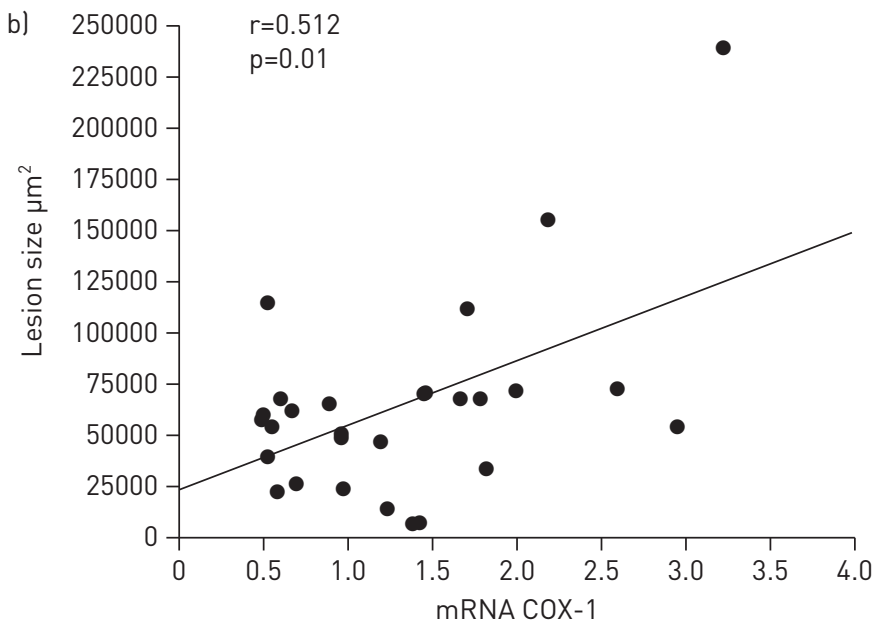

FIGURE 1 a) mRNA levels of COX-pathway genes in mice exposed to chronic intermittent hypoxia in the abdominal aorta. Regressions are shown between atherosclerotic lesion size and aortic mRNA levels of b) COX-1 and c) thromboxane synthase (TXBS). Data are expressed as fold-changes compared with normoxic mice (control). ${ }^{*}$ : $\mathrm{p}<0.05$ versus normoxic group. COX-1: cyclooxygenase type 1 isoform; COX-2: COX type 2 isoform; TXBS: thromboxane synthase; PGIS: prostacyclin synthase.

TABLE 2 Bodyweight, plasma cholesterol level, haematocrit and aortic prostanoid secretion of apolipoprotein E-deficient $\left(\mathrm{ApoE}^{-/-}\right)$mice exposed for 8 weeks to chronic intermittent hypoxia $(\mathrm{CIH})$ or normoxia, with treatment by $\mathrm{SC}-560$ or placebo during the last 4 weeks of exposure

\section{Normoxia}

\begin{tabular}{c}
\hline Placebo \\
10 \\
$28.8(25.8-31.0)$ \\
$14.2(9.13-20.2)$ \\
$44.5(42.5-45.0)$ \\
$55.2(35.3-94.7)$
\end{tabular}

778.3 (559.7-1059.1)

$13.4(9.9-21.6)$

ClH

\begin{tabular}{cc}
\hline Placebo & SC-560 \\
10 & 10 \\
$28.9(25.9-31.5)$ & $31.2(28.4-32.0)$ \\
$15.3(12.8-21.0)$ & $12.3(8.46-16.1)$ \\
$46.0(45.0-47.6)^{\#}$ & $45.5(43.5-48.5)$ \\
$56.7(44.0-80.8)$ & $7.86(2.65-11.86)^{+}$ \\
$802.1(638.3-1152.8)$ & $107.9(68.1-198.6)^{+}$ \\
$14.0(11.6-18.1)$ & $17.0(10.9-26.7)$ \\
\hline
\end{tabular}

Subjects $\mathrm{n}$

Bodyweight after exposure $\mathbf{g}$

Total cholesterol $\mathrm{mmol} \cdot \mathrm{L}^{-1}$

Haematocrit \%

Aortic secretion of $\mathrm{TXB}_{2}$

$\mathrm{ng} \cdot \mathrm{L}^{-1} \cdot \mathrm{mg}^{-1}$

Aortic secretion of 6-keto-PGF1 $\alpha$

$\mathrm{ng} \cdot \mathrm{L}^{-1} \cdot \mathrm{mg}^{-1}$

SC-560

$28.9(27.1-32.6)$

$11.1(8.44-17.6)$

$45.0(41.8-47.0)$

$3.13(1.80-22.6)^{\circ}$

$91.8(45.9-285.7)^{\circ}$

$24.1(9.3-40.8)$

Data are presented as median (10th-90th percentiles). TXB 2 : thromboxane; 6-keto-PGF1 $\alpha$ : 6-keto-prostaglandin F1 $\alpha .{ }^{*}: \mathrm{p}<0.05$ normoxic mice treated by placebo versus hypoxic mice treated by placebo; ${ }^{\circ}: \mathrm{p}<0.05$ normoxic mice treated by placebo versus normoxic mice treated by SC-560; ${ }^{+}: \mathrm{p}<0.05$ hypoxic mice treated by placebo versus hypoxic mice treated by SC-560. 

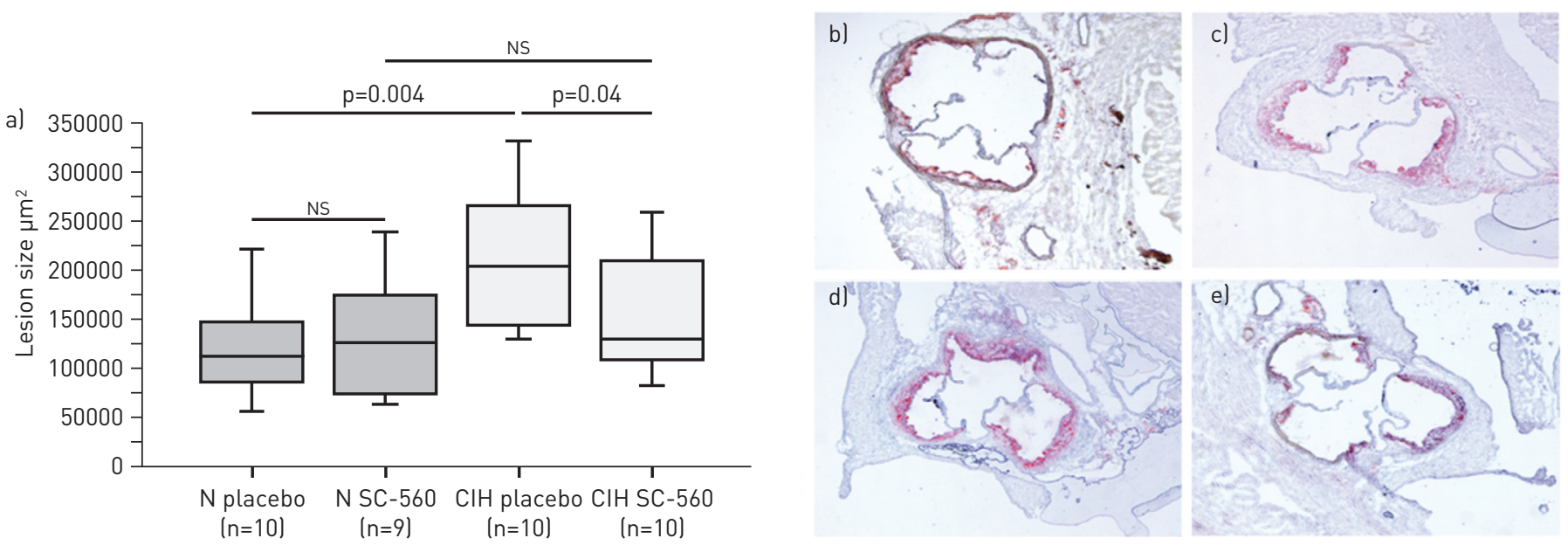

FIGURE 2 Effects of cyclooxygenase type 1 isoform (COX-1) inhibition on atherosclerosis in mice exposed to chronic intermittent hypoxia (CIH) or normoxia $(\mathrm{N})$. a) Lesion sizes. Data are presented as interquartile range (boxes), data range (whiskers) and median (horizontal line). Ns: nonsignificant. Representative photographs of Oil-Red-O staining for b) normoxia placebo, c) normoxia SC-560, d) CIH placebo and e) CIH SC-560.

TABLE 3 Baseline characteristics of controls and obstructive sleep apnoea (OSA) patients free of any known cardiovascular risk factor, matched for age and body mass index (BMI)

\section{Subjects $n$}

Males \%

BMI $\mathrm{kg} \cdot \mathrm{m}^{-2}$

Age years

Clinical systolic BP $\mathrm{mmHg}$

Clinical diastolic BP $\mathrm{mmHg}$

AHI events $\cdot h^{-1}$

RDI events $\cdot h^{-1}$

Minimal $\mathrm{SaO}_{2} \%$

$\mathrm{SaO}_{2}<90 \% \%$ TST

RAl events $\cdot h^{-1}$

Plasma glucose $\mathrm{mmol} \cdot \mathrm{L}^{-1}$

Plasma insulin $\mu \mathrm{UI} \cdot \mathrm{mL}^{-1}$

HOMA-IR index

Total cholesterol $\mathrm{mmol} \cdot \mathrm{L}^{-1}$

LDL cholesterol $\mathrm{mmol} \cdot \mathrm{L}^{-1}$

HDL cholesterol $\mathrm{mmol} \cdot \mathrm{L}^{-1}$

Triglycerides $\mathrm{mmol} \cdot \mathrm{L}^{-1}$

hsCRP $\mathrm{mg} \cdot \mathrm{L}^{-1}$

Right carotid IMT mm

Left carotid IMT mm

Mean carotid IMT mm

Overweight/normal BMI \%

Smoking \%

Hypertension \%

Metabolic syndrome \%

Dyslipidemia \%

Urinary 11-dTXB ${ }_{2}$

$\mathrm{pg} \cdot \mathrm{mg}^{-1}$ creatinine
Controls

OSA patients

25

76.0

$25.0(21.6-27.3)$

$51.2(42.7-62.6)$

$127(112-148)$

$85(76-97)$

$5.0(0.0-9.2)$

$6.6(1.0-15.7)$

$90.0(85.4-92.5)$

$0.00(0.00-1.00)$

$4.9(1.9-11.0)$

$4.7(4.3-5.3)$

$4.2(2.2-7.11)$

$0.86(0.50-1.68)$

$4.77(3.41-6.17)$

$2.76(1.57-3.92)$

$1.52(0.98-2.40)$

$0.89(0.52-1.90)$

$1.00(0.38-1.74)$

593 (515-653)

622 (527-780)

606 (523-710)

$48 / 52$

0

4

0

0

609.0 (265.8-945.3)

50

88.0
$24.9(21.8-27.9)$
$51.1(43.8-60.9)$
$125(110-146)$
$80(69-93)$
$35.4(14.9-59.4)$
$35.9(24.1-71.6)$
$84.5(75.3-90.0)$
$1.00(0.00-13.2)$
$28.0(12.0-49.1)$
$5.1(4.5-6.1)$
$5.48(2.85-8.57)$
$1.21(0.63-2.22)$
$5.68(4.41-6.62)$
$3.55(2.13-4.36)$
$1.52(1.10-2.13)$
$1.11(0.68-1.84)$
$0.95(0.30-3.30)$
$604(508-805)$
$647(515-872)$
$620(539-838)$
$46 / 56$
0
6
0
20

629.3 (266.4-933.1) p-value

0.20

0.83

0.99

0.72

0.29

$<0.0001$

$<0.0001$

$<0.0001$

$<0.0001$

$<0.0001$

0.03

0.11

0.13

0.004

0.003

0.94

0.07

0.61

0.44

0.32

0.52

0.87

1

1

1

$\mathbf{0 . 0 3}$

0.56

Data are presented as median (10th-90th percentiles), unless otherwise stated. BP: blood pressure; AHI: apnoea/hypopnoea index; RDI: respiratory disturbance index; $\mathrm{SaO}_{2}$ : arterial oxygen saturation; TST: total sleep time; RAl: respiratory arousal index; HOMA-IR: homeostasis model assessment of insulin resistance; LDL: low-density lipoprotein; HDL: high-density lipoprotein; hsCRP: high-sensitivity C reactive protein; IMT: intimamedia thickness; 11-dTXB 2 : 11-dehydrothromboxane. Bold indicates statistical significance. 
TABLE 4 Baseline characteristics of obstructive sleep apnoea (OSA) patients without (CVRF-) or with (CVRF+) associated cardiovascular risk factors

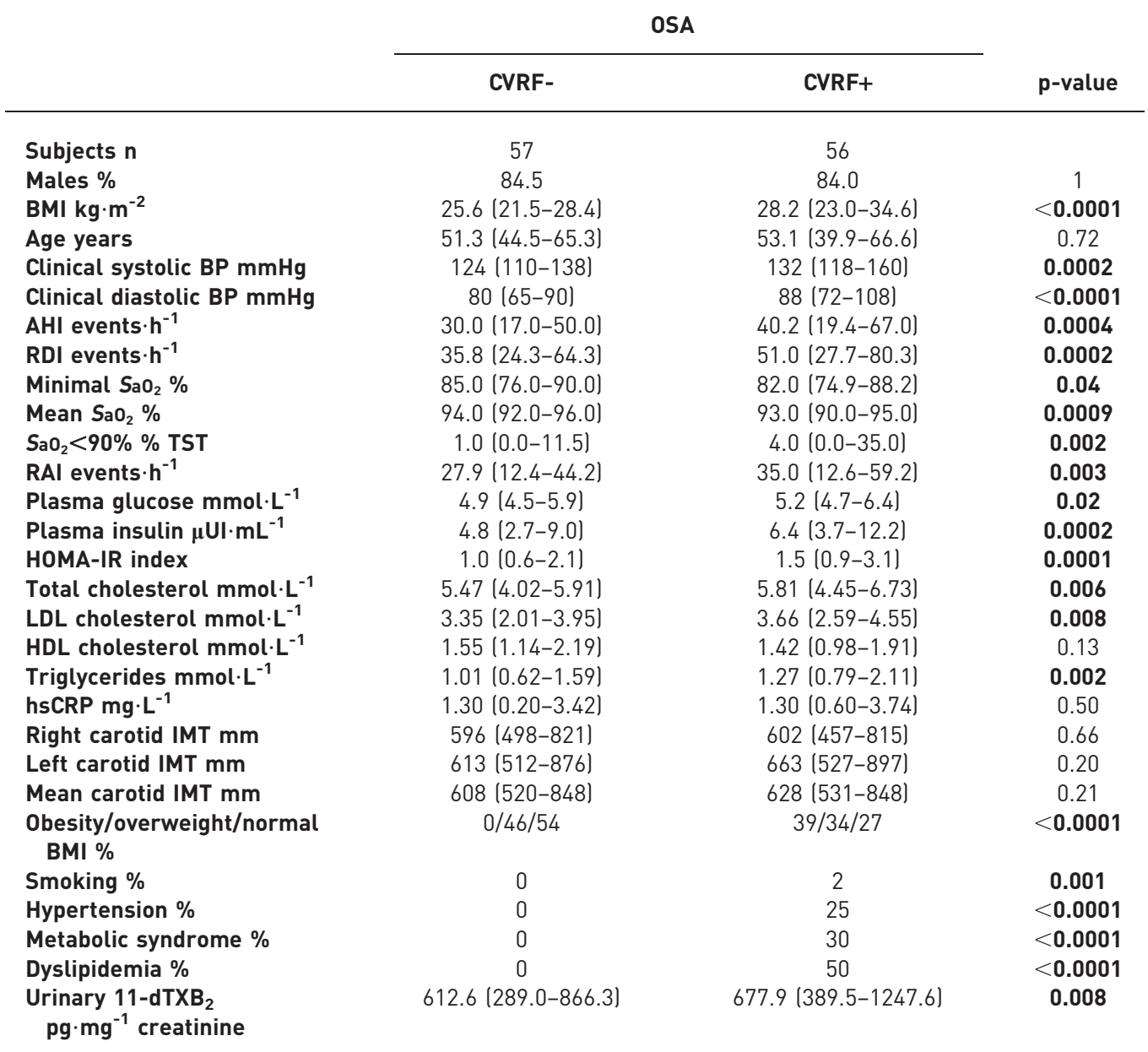

Data are presented as median (10th-90th percentiles), unless otherwise stated. BMI: body mass index; BP: blood pressure; $\mathrm{AHI}$ : apnoea/hypopnoea index; RDI: respiratory disturbance index; $\mathrm{SaO}_{2}$ : arterial oxygen saturation; TST: total sleep time; RAl: respiratory arousal index; HOMA-IR: homeostasis model assessment of insulin resistance; LDL: low-density lipoprotein; HDL: high-density lipoprotein; hsCRP: high-sensitivity C reactive protein; IMT: intima-media thickness; 11-dTXB 2 : 11-dehydrothromboxane. Bold indicates statistical significance.

\section{Clinical study}

11-dTXB ${ }_{2}$ and OSA

Baseline characteristics of the 25 controls and the 50 OSA patients matched for age and BMI are described in table 3. There was no significant difference for plasma insulin, hsCRP, HDL cholesterol, homeostatic model assessment insulin resistance index (HOMA-IR), BP, carotid intima-media thickness (IMT) and sex ratio. As expected, polysomnographic parameters were different between OSA and control groups. Plasma glucose, total and LDL cholesterol levels were significantly increased in OSA patients versus controls. Urinary 11- $\mathrm{dTXB}_{2}$ was not significantly different between OSA patients and controls (table 3 ).

\section{1-dTXB 2, OSA and cardiovascular risk factor}

Clinical, biological and polysomnographic parameters of OSA patients with or without CVRFs are shown in table 4. The OSA group with CVRFs had polysomnographic parameters, BP, BMI, plasma triglycerides, total and LDL cholesterol, glucose, insulin and HOMA-IR higher than the OSA group free of CVRFs (table 4). Conversely, these two groups were similar with regard to age, sex ratio, carotid IMT, HDL cholesterol and hsCRP (table 4). Urinary 11- $\mathrm{dTXB}_{2}$ was significantly increased in OSA patients with CVRFs compared with OSA patients free of CVRFs (fig. 3a). In OSA patients with or without CVRFs, 11-TXB 2 

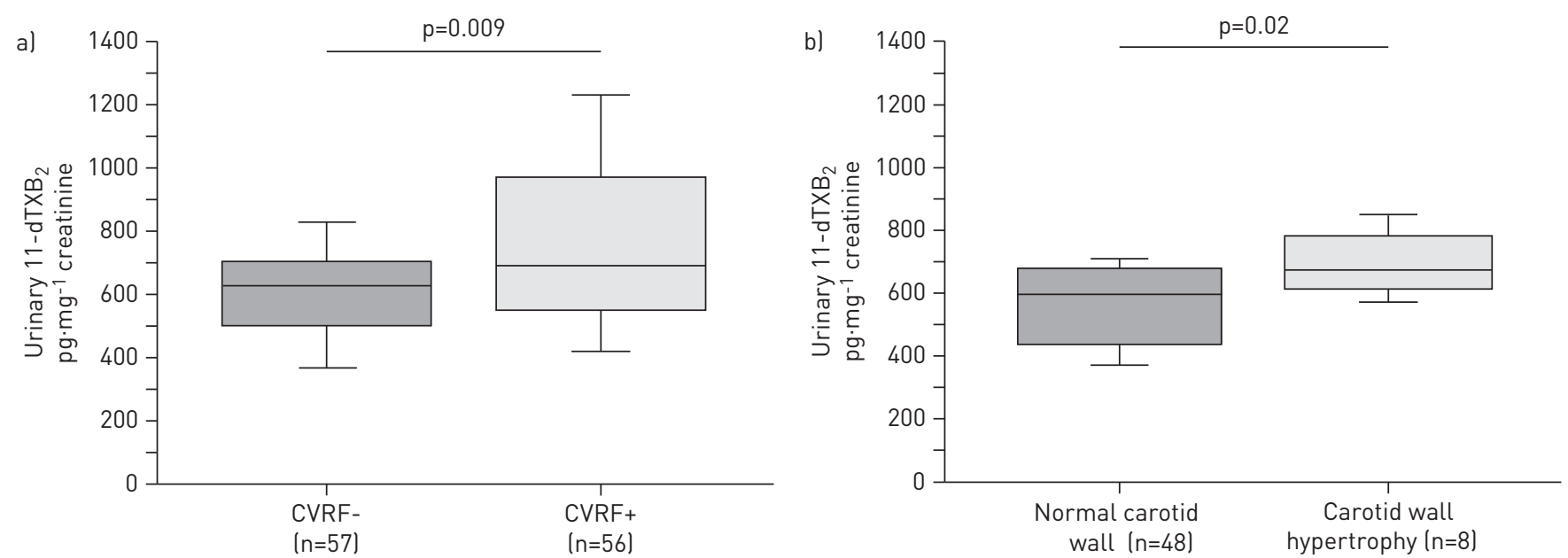

FIGURE 3 a) Urinary 11-dehydrothromboxane $\mathrm{B}_{2}\left(11-\mathrm{dTXB}_{2}\right)$ concentrations in obstructive sleep apnoea (OSA) patients with (CVRF+) and without (CVRF-) known cardiovascular risk factor. b) Urinary $11-\mathrm{dTXB}_{2}$ concentrations in OSA patients with or without carotid wall hypertrophy. Data are presented as interquartile range (boxes), data range (whiskers) and median (horizontal line).

levels were similar in patients with mild-to-moderate (AHI $<30$ events $\cdot \mathrm{h}^{-1}$ ) and severe OSA (AHI $\geqslant 30$ events $\left.\cdot \mathrm{h}^{-1}\right)($ data not shown).

To determine the CVRF involved in the increase of urinary $11-\mathrm{dTXB}_{2}$, simple regressions were performed. Hypertension $(r=0.190, p=0.05)$ and obesity $(r=0.242, p=0.01)$ were weakly associated with increased urinary excretion of $11-\mathrm{dTXB}_{2}$. In multiple regression model, obesity remained the sole independent predictive factor of urinary $11-\mathrm{dTXB}_{2}(\mathrm{r}=0.257, \mathrm{p}=0.04)$.

\section{Effect of CPAP treatment on urinary 11-dTXB 2 concentration}

CPAP treatment for at least 8 weeks significantly decreased AHI, RDI and respiratory arousal index, increased minimal nocturnal arterial oxygen saturation $\left(\mathrm{SaO}_{2}\right)$ and mean nocturnal $\mathrm{Sa}_{2} \mathrm{O}_{2}$, and decreased the percentage of time spent with a mean $\mathrm{SaO}_{2}<90 \%$. CPAP treatment induced no change on 11-dTXB 2 urinary concentration in OSA without or with CVRF (online supplementary tables S2 and S3).

\section{1-dTXB 2, OSA and vascular remodelling}

In OSA patients free of CVRF, vascular hypertrophy (defined by carotid IMT $>0.8 \mathrm{~mm}$ ) was associated with increased urinary $11-\mathrm{dTXB}_{2}$ concentrations compared with subjects without vascular hypertrophy $(\mathrm{p}=0.02)$ (fig. $3 \mathrm{~b})$.

\section{Discussion}

Our study demonstrated for the first time an activation of the COX pathway in $\mathrm{ApoE}^{-/-}$mice exposed to $\mathrm{CIH}$ and also in OSA patients with other CVRFs; this activation is associated with increased atherosclerotic lesions in mice and with early markers of atherosclerosis in OSA patients.

We showed that $\mathrm{CIH}$ mice had higher atherosclerotic lesions than normoxic air mice, suggesting that, in our model, $\mathrm{CIH}$ might have accelerated atherosclerosis development. This result is in accordance with previous works in $\mathrm{ApoE}^{-- \text {- }}$ mice exposed to $\mathrm{CIH}$ from 2 to 4 weeks $[4,5]$ or in $\mathrm{C} 57 \mathrm{BL} / 6 \mathrm{~J}$ mice exposed to $\mathrm{CIH}$ for 12 weeks [6].

In the present study, plasma cholesterol levels were not significantly different between normoxic air and $\mathrm{CIH}$ groups and atherosclerotic lesion sizes correlated with plasma cholesterol in normoxic air mice, but not in $\mathrm{CIH}$ mice. These data suggested that, in our model, $\mathrm{CIH}$-induced atherosclerosis might be independent of lipid disorders, and contrast with previous works showing that concomitant exposure to a high-fat highcholesterol diet and $\mathrm{CIH}$ aggravate both atherosclerosis and dyslipidemia in $\mathrm{ApoE}^{-/-}$mice [4] and in C57BL/ $6 \mathrm{~J}$ mice $[6,23]$. However, we recently showed that $\mathrm{CIH}$ also exerts pro-atherogenic effects through other contributing factors, notably the inflammatory process [5]. In agreement with this later hypothesis, our data showed an activation of the thromboxane pathway in CIH mice, as mRNA levels of COX-1 and TXBS were increased in aortic tissue of $\mathrm{CIH}$ mice. Furthermore, the correlation between these mRNA levels and atherosclerotic lesion size was consistent with direct effects of $\mathrm{TXA}_{2}$ on macrophages [24]. Unexpectedly, the aortic secretion of $\mathrm{TXB}_{2}$ and 6-keto-PGF1 $\alpha$, as well as the $\mathrm{TXB}_{2} / 6$-keto-PGF1 $\alpha$ ratio were similar in $\mathrm{CIH}$ and normoxic air mice. These results could be explained by the fact that we measured prostanoid secretion 
after aorta stimulation with A23187, and not the basal aortic production. A previous study performed in LDL r-knockout ( $\mathrm{r}-\mathrm{KO})$ mice demonstrated that the acceleration of atherogenesis in response to high fat diet was associated with increased basal levels of $\mathrm{TXB}_{2}$ and 6-keto-PGF1 $\alpha$ in the aortic arch [25], but measurements of prostanoids were performed by ELISA, which is not a specific method.

We chose to measure $\mathrm{TXB}_{2}$ and 6-keto-PGF1 $\alpha$ upon aorta stimulation with A23187 to obtain detectable prostanoid levels by our analytical technique (LC-MS/MS), which is highly specific but probably less sensitive. However, in our model, A23187 appears to stimulate $\mathrm{TXB}_{2}$ and 6-keto-PGF1 $\alpha$ release to the same extent, as both levels were highly correlated, which could explain the similar $\mathrm{TXB}_{2} / 6$-keto-PGF1 $\alpha$ ratio in aorta from $\mathrm{CIH}$ and normoxic air mice. We acknowledge that comparisons of basal $\mathrm{TXB}_{2}$ and 6-keto-PGF1 $\alpha$ production and Western blot analysis of COX-1 and TXBS would have been of interest with regard to the increased mRNA levels of COX-1 and TXBS induced by $\mathrm{CIH}$, and that their absence may represent a limitation of our study.

Moreover, treatment with the selective COX-1 inhibitor SC-560 during the last 4 weeks of CIH exposure reduced atherosclerosis progression in $\mathrm{CIH}$ mice, providing further evidence for a $\mathrm{CIH}$-dependent activation of the COX pathway. We already demonstrated that 4 weeks of $\mathrm{CIH}$ exposure were sufficient to induce atherosclerosis in $\mathrm{ApoE}^{-/-}$mice [5], thus it was of interest to explore the effects of COX-1 inhibition on established atherosclerotic lesions. As previously described [12], treatment with SC-560 had no effect on established atherosclerotic lesions in normoxic air mice, although it was effective in inhibiting COX-1 pathway as assessed by the measurement of aortic $\mathrm{TXB}_{2}$ and 6-keto-PGF1 $\alpha$ secretion. Collectively, these data demonstrated that in $\mathrm{ApoE}^{-/-}$mice, the atherogenic process was accelerated by $\mathrm{CIH}$ exposure, at least in part through COX-1 pathway activation. CIH activates leukocytes [26] and OSA patients display leukocyte activation $[7,8,27]$. By regulating the interaction between leukocytes, smooth muscle cells and endothelial cells, $\mathrm{TXA}_{2}$ promotes and $\mathrm{PGI}_{2}$ prevents the initiation and progression of atherogenesis [14].

To extend the thromboxane-pathway activation observed in $\mathrm{CIH}$ mice to OSA patients, we measured the urinary excretion of $11-\mathrm{dTXB}_{2}$, a validated biomarker of systemic $\mathrm{TXA}_{2}$ production [10]. Urinary 11- $\mathrm{dTXB}_{2}$ concentrations of OSA patients free of CVRF were not different to those of controls carefully matched for age and BMI, two major confounding factors often present in studies focused on the underlying inflammation associated to OSA. These data suggested that OSA itself was not associated with an increased urinary 11- $\mathrm{dTXB}_{2}$ excretion, an hypothesis confirmed by the observation that CPAP treatment had no influence on urinary $11-\mathrm{dTXB}_{2}$ level. However, increased urinary $11-\mathrm{dTXB}_{2}$ concentrations have previously been described in patients with cardiovascular diseases including obesity [28] and hypertension [29]. Consistent with these data, we showed that OSA patients with an associated CVRF had higher urinary concentrations of $11-\mathrm{dTXB}_{2}$ than OSA patients free of a known CVRF. Furthermore, among the studied CVRFs, obesity was the sole independent predictor of urinary $11-\mathrm{dTXB}_{2}$ excretion, which is in agreement with a previous study demonstrating an increased urinary $11-\mathrm{dTXB}_{2}$ excretion in obese females [28]. This result provided further evidence for the major role of obesity in arachidonic acid metabolism activation in OSA patients, as we previously described for the 5-lipooxygenase pathway [9]. Finally, urinary 11-dTXB 2 excretion was associated with carotid wall hypertrophy in OSA patients. Again, these findings were consistent with the proliferative effects of $\mathrm{TXA}_{2}$ on vascular smooth muscle cells [10], although it must be kept in mind that vascular remodelling is a complex process implying many mediators and that COX pathway activation might not be the sole mechanism of vascular remodelling in OSA patients with CVRFs.

In conclusion, our study showed an activation of the COX-1 pathway in $\mathrm{ApoE}^{-/-}$mice exposed to $\mathrm{CIH}$, which contributed to the acceleration of the atherogenic process induced by this stimulus. Such an activation of the COX-1 pathway was also found in OSA patients with associated CVRFs. This study extends the activation of the arachidonic acid metabolism previously described for the leukotriene pathway $[7,9]$ to the COX-1 pathway in OSA in relation to vascular remodelling. These findings open the field to the interest of new pharmacological approaches (dual COX and 5-lipoxygenase inhibitor [30]) in the prevention of cardiovascular morbidity in OSA patients.

\section{Acknowledgements}

The authors thank Nathalie Arnold (CHU, Hôpital A. Michallon, Pôle réeducation et Physiologie, BP217, Grenoble, France) for statistical analyses, Jean-François Jourdil, Karine Scalabrino, Cécile Girard (CHU, Hôpital A. Michallon, Laboratoire de Pharmacologie, BP217, Grenoble) and Sandrine Cachot (INSERM, U1042, Grenoble) for expert technical assistance.

\section{References}

Dematteis M, Godin-Ribuot D, Arnaud C, et al. Cardiovascular consequences of sleep-disordered breathing: contribution of animal models to understanding the human disease. ILAR J 2009; 50: 262-281.

2 Baguet JP, Hammer L, Lévy P, et al. The severity of oxygen desaturation is predictive of carotid wall thickening and plaque occurrence. Chest 2005; 128: 3407-3412. 
3 Somers VK, White DP, Amin R, et al. Sleep apnea and cardiovascular disease: an American Heart Association/ American College of Cardiology Foundation Scientific Statement from the American Heart Association Council for High Blood Pressure Research Professional Education Committee, Council on Clinical Cardiology, Stroke Council, and Council on Cardiovascular Nursing. J Am Coll Cardiol 2008; 52: 686-717.

4 Jun J, Reinke C, Bedja D, et al. Effect of intermittent hypoxia on atherosclerosis in apolipoprotein E-deficient mice. Atherosclerosis 2010; 209: 381-386.

5 Arnaud C, Poulain L, Lévy P, et al. Inflammation contributes to the atherogenic role of intermittent hypoxia in apolipoprotein-E knock out mice. Atherosclerosis 2011; 219: 425-431.

6 Savransky V, Nanayakkara A, Li J, et al. Chronic intermittent hypoxia induces atherosclerosis. Am J Respir Crit Care Med 2007; 175: 1290-1297.

7 Lefebvre B, Pépin JL, Baguet JP, et al. Leukotriene B4: early mediator of atherosclerosis in obstructive sleep apnoea? Eur Respir J 2008; 32: 113-120.

8 Stanke-Labesque F, Pepin JL, de Jouvencel T, et al. Leukotriene B4 pathway activation and atherosclerosis in obstructive sleep apnea. J Lipid Res 2012; 53: 1944-1951.

9 Stanke-Labesque F, Bäck M, Lefebvre B, et al. Increased urinary leukotriene E4 excretion in obstructive sleep apnea: effects of obesity and hypoxia. J Allergy Clin Immunol 2009; 124: 364-370.

10 Capra V, Bäck M, Barbieri SS, et al. Eicosanoids and their drugs in cardiovascular diseases: focus on atherosclerosis and stroke. Med Res Rev 2013; 33: 364-438.

11 Cyrus T, Sung S, Zhao L, et al. Effect of low-dose aspirin on vascular inflammation, plaque stability, and atherogenesis in low-density lipoprotein receptor-deficient mice. Circulation 2002; 106: 1282-1287.

12 Belton OA, Duffy A, Toomey S, et al. Cyclooxygenase isoforms and platelet vessel wall interactions in the apolipoprotein E knockout mouse model of atherosclerosis. Circulation 2003; 108: 3017-3023.

13 McClelland S, Gawaz M, Kennerknecht E, et al. Contribution of cyclooxygenase-1 to thromboxane formation, platelet-vessel wall interactions and atherosclerosis in the ApoE null mouse. Atherosclerosis 2009; 202: 84-91.

14 Kobayashi T, Tahara Y, Matsumoto M, et al. Roles of thromboxane A(2) and prostacyclin in the development of atherosclerosis in apoE-deficient mice. J Clin Invest 2004; 114: 784-794.

15 Montine TJ, Sonnen JA, Milne G, et al. Elevated ratio of urinary metabolites of thromboxane and prostacyclin is associated with adverse cardiovascular events in ADAPT. PLoS One 2010; 5: e9340.

16 Bäck M, Yin L, Ingelsson E. Cyclooxygenase-2 inhibitors and cardiovascular risk in a nation-wide cohort study after the withdrawal of rofecoxib. Eur Heart J 2012; 33: 1928-1933.

17 Krieger J, Benzoni D, Sforza E, et al. Urinary excretion of prostanoids during sleep in obstructive sleep apnoea patients. Clin Exp Pharmacol Physiol 1991; 18: 551-555.

18 Kimura H, Niijima M, Abe Y, et al. Compensatory excretion of prostacyclin and thromboxane metabolites in obstructive sleep apnea syndrome. Intern Med 1998; 37: 127-133.

19 Bäck M, Sultan A, Ovchinnikova O, et al. 5-Lipoxygenase-activating protein: a potential link between innate and adaptive immunity in atherosclerosis and adipose tissue inflammation. Circ Res 2007; 100: 946-949.

20 Holt RI. International Diabetes Federation re-defines the metabolic syndrome. Diabetes Obes Metab 2005; 7: 618-620.

21 Hosselet J, Ayappa I, Norman RG, et al. Classification of sleep-disordered breathing. Am J Respir Crit Care Med 2001; 163: 398-405.

22 Casetta B, Vecchione G, Tomaiuolo M, et al. Setting up a 2D-LC/MS/MS method for the rapid quantitation of the prostanoid metabolites 6-oxo-PGF $(1 \alpha)$ and TXB2 as markers for hemostasis assessment. J Mass Spectrom 2009; 44: $346-352$.

23 Li J, Thorne LN, Punjabi NM, et al. Intermittent hypoxia induces hyperlipidemia in lean mice. Circ Res 2005; 97: 698-706.

24 Caughey GE, Pouliot M, Cleland LG, et al. Regulation of tumor necrosis factor- $\alpha$ and IL- $1 \beta$ synthesis by thromboxane A2 in nonadherent human monocytes. J Immunol 1997; 158: 351-358.

25 Cyrus T, Ding T, Praticò D. Expression of thromboxane synthase, prostacyclin synthase and thromboxane receptor in atherosclerotic lesions: correlation with plaque composition. Atherosclerosis 2010; 208: 376-381.

26 Arnaud C, Beguin PC, Lantuejoul S, et al. The inflammatory preatherosclerotic remodeling induced by intermittent hypoxia is attenuated by RANTES/CCL5 inhibition. Am J Respir Crit Care Med 2011; 184: 724-731.

27 Lavie L. Oxidative stress inflammation and endothelial dysfunction in obstructive sleep apnea. Front Biosci (Elite Ed) 2012; 4: 1391-1403.

28 Davi G, Guagnano MT, Ciabattoni G, et al. Platelet activation in obese women: role of inflammation and oxidant stress. JAMA 2002; 288: 2008-2014.

29 Minuz P, Patrignani P, Gaino S, et al. Determinants of platelet activation in human essential hypertension. Hypertension 2004; 43: 64-70.

30 Choi JH, Jeon HJ, Park JG, et al. Anti-atherogenic effect of BHB-TZD having inhibitory activities on cyclooxygenase and 5-lipoxygenase in hyperlipidemic mice. Atherosclerosis 2010; 212: 146-152. 